\title{
An assessment of the PTT model on the impacting drop problem
}

\author{
M. F. Tomé ${ }^{3} \cdot$ R. Merejolli ${ }^{1}$ - G. S. Paulo ${ }^{2}$ S. McKee $^{4}$ \\ Received: 7 April 2018/Accepted: 28 August 2018/Published online: 5 September 2018 \\ (C) The Brazilian Society of Mechanical Sciences and Engineering 2018
}

\begin{abstract}
This work presents a numerical method and simulations of axisymmetric free-surface flows of a polymeric fluid using the Phan-Thien-Tanner (PTT) constitutive relationship. The governing equations are solved by finite differences on a staggered grid that employs accurate boundary conditions on the free surface together with bounded, monotone, high-order upwinding approximation of the convection terms. The numerical method is partially verified by solving fully developed flow in a tube and comparing it to a known analytic solution. The problem of a drop impacting on a rigid plate is considered. The effect of varying the five parameters, characterizing the PTT model, is studied extensively, and the results are interpreted physically. The expansion-contraction phenomenon of the viscoelastic drop that occurs when it hits a flat surface is tracked, and the time evolution of the normal and shear components of the extra-stress tensor is analyzed. To further verify the code, the impacting drop of an Oldroyd-B fluid is simulated and the time evolution of the drop width is compared to that of other authors.
\end{abstract}

Keywords PTT model · Axisymmetric flow · Free-surface flow · Impacting drop · Finite difference method

\section{Introduction}

The rheology of polymeric solutions is dependent on the molecular architecture of the constituent molecules: chain stiffness and chain branching and, of course, molecular weight. Polymeric solutions also depend, of course, upon the amount of Newtonian solvent, and any polymeric solution is composed of polymers of different lengths. Thus, the development of a satisfactory constitutive relationship is a difficult task. Despite this, many models have been proposed, see, for example, [1] where a comprehensive list, up to that date at least, may be found. Except occasionally in the simplest of geometries, analytic

Technical Editor: Cezar Negrao.

M. F. Tomé

murilo@icmc.usp.br

1 Department of Mathematics, Federal University of Mato Grosso do Sul, Ponta Porã, Brazil

2 São Paulo State University (Unesp), School of Technology and Sciences, Presidente Prudente, Brazil

3 Department of Applied Mathematics and Statistics, University of São Paulo, São Carlos, Brazil

4 Department of Mathematics and Statistics, University of Strathclyde, Glasgow, UK solutions cannot be found and consequently most researchers resort to numerical methods (see, e.g., [2]).

The better known constitutive models that have been studied include Oldroyd-B [3-6], Phan-Thien-Tanner (PTT) [7-10], FENE-CR [11-13], XPP [10, 14, 15], Giesekus $[15,16]$, among others. Within the class of differential models, the PTT constitutive equation [7] is known to provide a reasonable fit to polymeric solutions and polymer melts and has attracted the attention of several scientists (e.g., [5, 9, 17-20]).

This paper examines the flow produced by a drop as it impinges on a rigid surface: the so-called impacting drop. Several authors have attempted to solve this problem: Oishi et al. [14] employed the XPP model using finite differences while $\mathrm{Xu}$ et al. [21] simulated the same problem using the Oldroyd-B and the Upper Convected Maxwell models in three dimensions with an improved smoothed particle hydrodynamics method (SPH). More recently, Figueiredo et al. [22] investigated the drop impact problem employing the Giesekus and XPP models using a three-dimensional finite difference code based on the marker-and-cell (MAC) methodology and compared their Oldroyd-B results with the data obtained by $\mathrm{Xu}$ et al. [21] together with results from the OpenFOAM ${ }^{\circledR}$ [23] code.

With regard to free-surface flows, there would appear to be few authors (but see $[17,24,25]$ ) who have considered 
solving the PTT model. The aim of this paper is to provide a numerical method for solving the PTT equation, to demonstrate that it converges and agrees with an analytic solution for simple pipe flow and, by solving the impacting drop problem, to study in detail the effect of varying the five parameters that characterize the complete PTT model. Where possible, comparisons with results from the literature are provided.

This work is organized as follows: The impacting drop problem is described in Sect. 2, and Sect. 3 deals with the methodology; in Sect. 4, a numerical computation of tube filling is compared with a known analytic solution and convergence results using mesh refinement are provided. Section 5 simulates the impacting drop and effects a comparison with existing data; mesh refinement is undertaken, and a sensitivity analysis of the parameters characterizing the PTT constitutive relationship is performed. Conclusions are summarized in Sect. 6.

\section{The drop impacting problem}

A spherical drop of diameter $d=2 R$ is placed at a height $H$ above a rigid plate (see Fig. 1a). The flow is assumed to be axisymmetric so the computational domain is displayed in Fig. 1b. At $t=0$, the drop starts flowing downwards with initial velocity $v(r, h, 0)=-U$ and $u=0$ while the components of the extra-stress tensor $\tau(r, h, 0)$ are set to zero. After the drop has impacted the plate, it is anticipated that it will flow radially expanding and, or contracting its diameter $\mathrm{d}(t)$. Viscous, inertial, gravitational and elastic forces can affect the flow after the drop has impacted the plate. The surrounding air is supposed to be quiescent, and surface tension forces are neglected since they are assumed to be small.

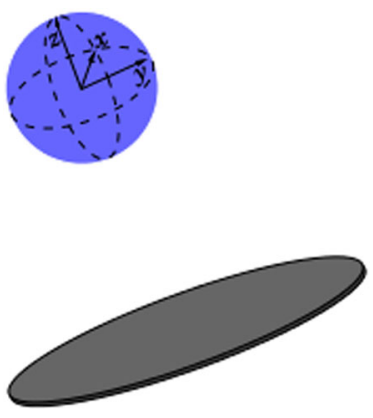

(a)

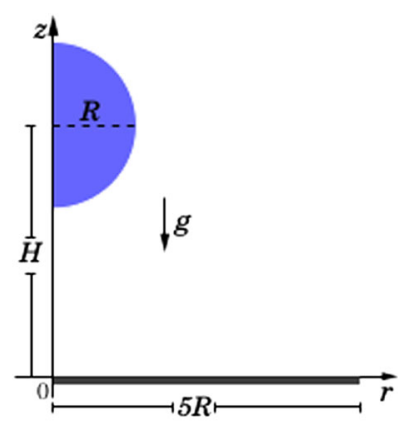

(b)
Fig. 1 Simulation of drop impacting. a 3D view of initial state; b computational domain employed

\section{Methodology}

The governing equations for incompressible flows are described by the mass conservation and momentum equations coupled with a constitutive relationship characterizing the PTT model [7]. In this work, the extra-stress tensor $\tau$ is split as a sum of a Newtonian tensor $\left(\eta_{S} \mathbf{D}\right)$ and a nonNewtonian tensor $(\mathbf{S})$ that are defined by

$\tau^{*}=2 \eta_{S} \mathbf{D}^{*}+\mathbf{S}^{*}, \quad \mathbf{D}^{*}=\frac{1}{2}\left[\nabla \mathbf{u}^{*}+\left(\nabla \mathbf{u}^{*}\right)^{\mathrm{T}}\right]$

where $\eta_{S}$ represents a Newtonian viscosity (called solvent viscosity); the non-Newtonian tensor $\mathbf{S}$ is responsible for the effects of elasticity in the flow and is obtained from the solution of the PTT constitutive equation. With this splitting, the governing equations can be written in conservative nondimensional form as,

$$
\begin{aligned}
& \begin{aligned}
\nabla \cdot \mathbf{u}= & 0, \\
\frac{\partial \mathbf{u}}{\partial t}= & -\nabla \cdot\left(\mathbf{u u}^{\mathrm{T}}\right)-\nabla p+\frac{\beta}{\operatorname{Re}} \nabla^{2} \mathbf{u}+\nabla \cdot \mathbf{S} \\
& +\frac{1}{F r^{2}} \mathbf{g}, \\
\frac{\partial \mathbf{S}}{\partial t}+\nabla \cdot(\mathbf{u S})= & -\left[\frac{1+\varepsilon \operatorname{Re} W i(\operatorname{tr}(\mathbf{S}))}{W i}\right] \mathbf{S} \\
& +\left[\mathbf{S}(\nabla \mathbf{u}-\xi \mathbf{D})+(\nabla \mathbf{u}-\xi \mathbf{D})^{T} \mathbf{S}\right] \\
& +\frac{(1-\beta)}{\operatorname{Re} W i} \mathbf{D},
\end{aligned}
\end{aligned}
$$

where $t$ is the time, $\mathbf{u}$ is the velocity vector, $p$ is the pressure, $\mathbf{S}$ is the PTT extra-stress tensor and $\mathbf{g}$ is the gravitational acceleration. The nondimensional numbers $F r=\frac{U}{\sqrt{g L}}, R e=\frac{\rho U L}{\eta_{0}}, W i=\lambda \frac{U}{L}$ are, respectively, the Froude, Reynolds and Weissenberg numbers. The constants, $L, U$ and $\rho$ denote typical scalings for length, velocity and density, respectively; the parameter $\lambda$ is the fluid relaxation time. To obtain the nondimensional Eqs. (2)-(4), the following nondimensional variables were employed:

$$
\begin{aligned}
t & =\frac{U}{L} t^{*}, \quad \mathbf{x}=\frac{\mathbf{x}^{*}}{L}, \quad \mathbf{u}=\frac{\mathbf{u}^{*}}{U}, \quad p=\frac{p^{*}}{\rho U^{2}}, \\
\mathbf{S} & =\frac{\mathbf{S}^{*}}{\rho U^{2}}, \quad \mathbf{D}=\frac{U}{L} \mathbf{D}^{*},
\end{aligned}
$$

where $\mathbf{x}=(r, z)$. The amount of Newtonian solvent is controlled by the dimensionless coefficient $\beta=\frac{\eta_{S}}{\eta_{0}}$, where $\eta_{0}=\eta_{S}+\eta_{P}$ is the total viscosity at zero shear while $\eta_{S}$ and $\eta_{P}$ represent the Newtonian and polymeric viscosities, respectively. The model parameters $\varepsilon$ and $\xi$ have values in the interval $[0,1]$. When $\varepsilon=\xi=0$, the PTT reduces to the Oldroyd-B model. 
Equations (1)-(4) are solved using cylindrical coordinates $\mathrm{Orz}$ for axisymmetric flows. The cross-stream (radial) coordinate and velocity are represented by $r$ and $u$ while the streamwise coordinate and velocity are denoted by $z$ and $v$, respectively. Thus, the velocity and the non-Newtonian extra-stress tensor are represented by

$$
\begin{aligned}
\mathbf{u}(r, z, t) & =\left[\begin{array}{c}
u(r, z, t) \\
0 \\
v(r, z, t)
\end{array}\right] \text { and } \\
\mathbf{S}(r, z, t) & =\left[\begin{array}{ccc}
S^{r r}(r, z, t) & 0 & S^{r z}(r, z, t) \\
0 & S^{\theta \theta} & 0 \\
S^{r z}(r, z, t) & 0 & S^{z z}(r, z, t)
\end{array}\right] .
\end{aligned}
$$

The coordinate system is centered on the symmetry axis which is positioned in the vertical direction at $r=0$. To solve the system of Eqs. (1)-(4), we consider all variables initially to be zero. At fluid entrances (inflows), unless otherwise stated, the velocity is specified by $\mathbf{u}=\mathbf{U}_{\text {inf }}$ and the non-Newtonian extra-stress tensor is set as $\mathbf{S}=\mathbf{S}_{\text {inf }}$. At fluid exits (outflows), homogeneous Neumann conditions are imposed, namely $\frac{\partial \mathbf{u}}{\partial z}=0$ and $\frac{\partial \mathbf{S}}{\partial z}=0$. On rigid boundaries, the velocity obeys the no-slip condition $(\mathbf{u}=\mathbf{0})$. We use this condition together with the continuity equation Eq. (2) to simplify Eq. (4) on rigid boundaries and solve the simplified equation to obtain the tensor $\mathbf{S}$ on rigid boundaries. On the symmetry axis, the velocity satisfies the so-called free-slip condition $\left(u=\frac{\partial v}{\partial r}=0\right.$ on $\left.r=0\right)$, and following a similar procedure used for the rigid boundaries, Eq. (4) is simplified and solved numerically to obtain each component of the tensor $\mathbf{S}$ on the symmetry axis.

We consider unsteady free-surface flows of a fluid moving into a passive atmosphere which is assumed to be at zero pressure. In the absence of surface tension, the normal and tangential components of the stress tensor must be continuous across any free surface, so that on that surface we must have (see [26])

$\mathbf{n}^{T} \cdot \boldsymbol{\sigma} \cdot \mathbf{n}=0 \quad$ and $\quad \mathbf{m}^{T} \cdot \boldsymbol{\sigma} \cdot \mathbf{n}=0$,

where $\mathbf{n}=\left(n_{r}, 0, n_{z}\right)^{\mathrm{T}}$ and $\mathbf{m}=\left(m_{r}, 0, m_{z}\right)^{\mathrm{T}}$ denote unit normal and tangential vectors to the surface, respectively, and

$\boldsymbol{\sigma}=-p \boldsymbol{I}+\frac{\beta}{R e}\left(\nabla \mathbf{u}+(\nabla \mathbf{u})^{T}\right)+\mathbf{S}$

is the stress tensor. By taking $\mathbf{m}=\left(n_{z}, 0,-n_{r}\right)^{T}$, the stress conditions (5) can be written as

$$
\begin{gathered}
p=\frac{2 \beta}{R e}\left[\frac{\partial u}{\partial r} n_{r}^{2}+\left(\frac{\partial u}{\partial z}+\frac{\partial v}{\partial r}\right) n_{r} n_{z}+\frac{\partial v}{\partial z} n_{z}^{2}\right] \\
+S^{r r} n_{r}^{2}+2 S^{z r} n_{r} n_{z}+S^{z z} n_{z}^{2}, \\
2\left(\frac{\partial v}{\partial z}-\frac{\partial u}{\partial r}\right) n_{r} n_{z}+\left(\frac{\partial u}{\partial z}+\frac{\partial v}{\partial r}\right)\left(n_{r}^{2}-n_{z}^{2}\right) \\
=\frac{R e}{\beta}\left(S^{r r}-S^{z z}\right) n_{r} n_{z}+S^{r z}\left(n_{z}^{2}-n_{r}^{2}\right) .
\end{gathered}
$$

In the absence of surface tension, equations (7) and (8) represent the appropriate boundary conditions on a fluid free surface (see [26]).

In this work, the numerical methodology employed is an extension of the technique introduced by Tomé et al. [6] which solved incompressible isothermal axisymmetric flows of an Oldroyd-B fluid. The GENSMAC (GENeralized Simplified Marker-And-Cell) [27] methodology is used to solve the time-dependent momentum and mass conservation equations on a staggered grid (see Fig. 2a). We are concerned with time-dependent moving free
Fig. 2 Staggered grid employed (a); type of cells within the mesh (b)

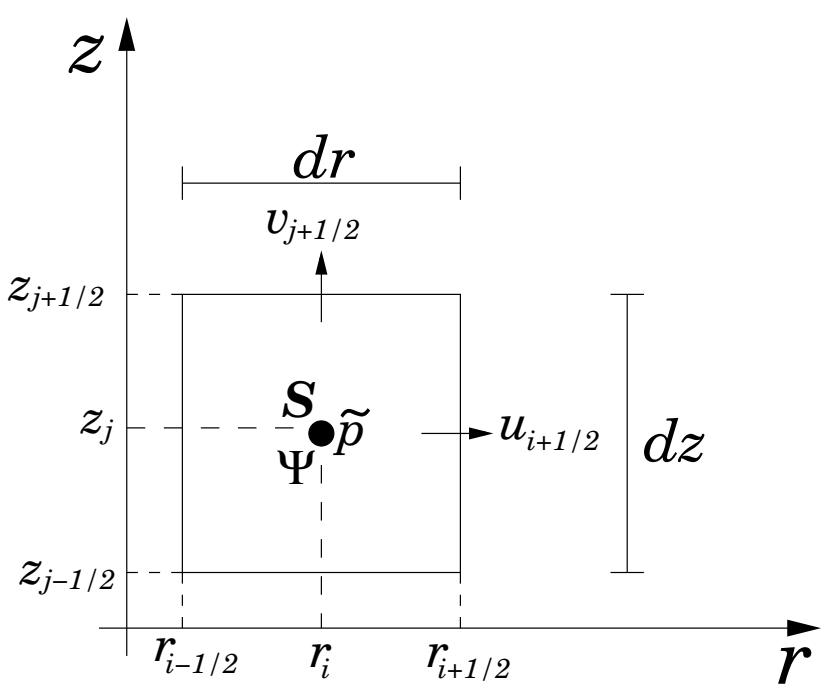

(a)

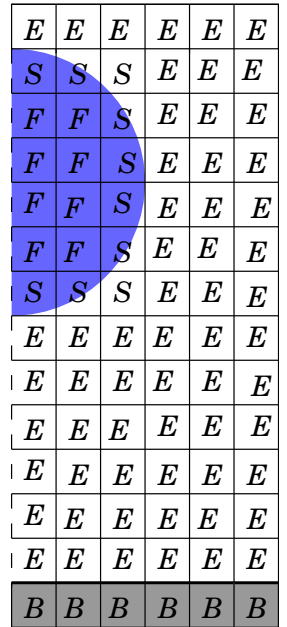

(b) 
surfaces so that a technique for identifying the fluid free surface is required. For this, the cells within the mesh are classified as: B-cells (cells that define rigid boundaries); I-cells (cells that define inflow boundaries); O-cells (cells that define outflow boundaries); F-cells (cells that contain fluid - the governing equations are solved in these cells); $\mathrm{S}$-cells (cells that define the free surface-the governing equations are solved in these cells together with the freesurface boundary conditions given by equations (7) and (8)); and E-cells (cells that do not contain fluid). Figure $2 b$ displays these type of cells during a calculational cycle. More details about the classification of cells and discretization of the equations at the free surface can be found in $[6,27,28]$. The fluid is described by a set of massless particles that moves in time with the updated velocity field. The visualization of the fluid surface is provided by joining these particles by straight lines (see Fig. 3); the respective volume of fluid is defined by the surface area occupied by the closed surface (see blue area shown in Fig. 3).

The projection method is applied to decouple the momentum and the mass conservations equations via the Helmholtz-Hodge decomposition theorem (HHDT) [29]. Both the momentum and the PTT equations are solved by a finite difference method that approximates the time derivative by the explicit Euler method while the spatial derivatives are approximated by second-order differences. The convection terms in the momentum and PTT constitutive equations are computed by the high-order upwind method, CUBISTA [5]. The algorithm for calculating $\mathbf{u}, p$ and $\mathbf{S}$ can be summarized as follows.

It is supposed that $\mathbf{u}\left(\mathbf{x}, t_{n}\right)=\mathbf{u}^{(n)}, \mathbf{S}\left(\mathbf{x}, t_{n}\right)=\mathbf{S}^{(n)}$ and $p\left(\mathbf{x}, t_{n}\right)=p^{(n)}$ are known. Then, $\mathbf{u}\left(\mathbf{x}, t_{n+1}\right)=\mathbf{u}^{(n+1)}$, $\mathbf{S}\left(\mathbf{x}, t_{n+1}\right)=\mathbf{S}^{(n+1)}$ and $p\left(\mathbf{x}, t_{n+1}\right)=p^{(n+1)}$ are computed in the following steps:



Fig. 3 Particles defining the fluid and volume of fluid (blue area)
1. Set up the boundary conditions for $\mathbf{u}$ and $\mathbf{S}$ and compute a pressure field $\widetilde{p}$ that satisfies condition (7) on the free surface. Usually, $\widetilde{p}=p^{(n)}$ in F-cells while in S-cells $\widetilde{p}$ is calculated by the pressure equation (7).

2. Compute $\widetilde{\mathbf{u}}^{(n+1)}$ by

$$
\begin{aligned}
\widetilde{\mathbf{u}}^{(n+1)}= & \mathbf{u}^{(n)}+\delta t\left\{-\nabla \cdot\left(\left(\mathbf{u u}^{\mathrm{T}}\right)^{(n)}\right)-\nabla \widetilde{p}\right. \\
& \left.+\frac{\beta}{\operatorname{Re}} \nabla^{2} \mathbf{u}^{(n)}+\nabla \cdot \mathbf{S}^{(n)}+\frac{1}{F r^{2}} \mathbf{g}\right\} .
\end{aligned}
$$

by setting for $\widetilde{\mathbf{u}}$ the same values of $\mathbf{u}$ at the boundaries.

3. Solve the Poisson equation

$\nabla^{2} \psi^{(n+1)}=\nabla \cdot \widetilde{\mathbf{u}}^{(n+1)}$,

subject to homogeneous Neumann conditions on inflows, rigid boundaries and the symmetry axis, and $\psi=0$ on outflows and free surfaces, since the Helmholtz-Hodge decomposition requires that $\nabla \psi=$ $(\widetilde{\mathbf{u}}-\mathbf{u})$ within the domain.

4. Calculate the final velocity field

$\mathbf{u}^{(n+1)}=\widetilde{\mathbf{u}}^{(n+1)}-\nabla \psi^{(n+1)}$

5. Compute the pressure

$p^{(n+1)}=\widetilde{p}+\frac{\psi^{(n+1)}}{\delta t}$

6. Calculate the tensor $\mathbf{S}^{(n+1)}$ by solving

$$
\begin{aligned}
\mathbf{D}^{(n+1)}= & \frac{1}{2}\left[\nabla\left(\mathbf{u}^{(n+1)}\right)+\left(\nabla\left(\mathbf{u}^{(n+1)}\right)\right)^{\mathrm{T}}\right] \\
\mathbf{S}^{(n+1)}= & \mathbf{S}^{(n)}+\delta t\left\{\nabla \cdot\left(\mathbf{u}^{(n+1)} \mathbf{S}^{(n)}\right)\right. \\
& -\left[\frac{1+\varepsilon \operatorname{ReWi}\left(\operatorname{tr}\left(\mathbf{S}^{(n)}\right)\right)}{W i}\right] \mathbf{S}^{(n)} \\
& +\left[\mathbf{S}^{(n)}\left(\nabla \mathbf{u}^{(n+1)}-\xi \mathbf{D}^{(n+1)}\right)\right. \\
& \left.+\left(\nabla \mathbf{u}^{(n+1)}-\xi \mathbf{D}^{(n+1)}\right)^{T} \mathbf{S}^{(n)}\right] \\
& +\frac{(1-\beta)}{\operatorname{Re} W i} \mathbf{D}^{(n+1)} .
\end{aligned}
$$

7. The last step is to update the positions of the particles by solving the system of ordinary differential equations:

$\frac{\mathrm{d} \mathbf{x}}{\mathrm{d} t}=\mathbf{u}^{(n+1)}$

using the second-order RK21 scheme as proposed by Oishi et al. [14]. 
The details of the finite difference equations for solving steps 1-7 are provided in the papers of Tomé et al. [6, 30]. The cylindrical coordinates version of the mass conservation, momentum conservation and PTT constitutive equations are displayed in "Appendix 1."

The explicit computation of the momentum equation imposes the following limitations on the time-step size $(\delta t)$ :

(i) $\delta t_{r} \leq \frac{\delta r}{|u|_{\max }}, \quad \delta t_{z} \leq \frac{\delta z}{|v|_{\max }}$,

(ii) $\delta t_{\text {visc }} \leq \frac{\operatorname{Re}}{2}\left(\frac{1}{(\delta r)^{2}}+\frac{1}{(\delta z)^{2}}\right)^{-1}$,

where $|u|_{\max },|v|_{\max }$ are the maximum of the velocity field at time $t$. Condition (i) is essentially the CFL condition while restriction (ii) results from a von Neumann analysis applied to the linearized equations. The selected time step is

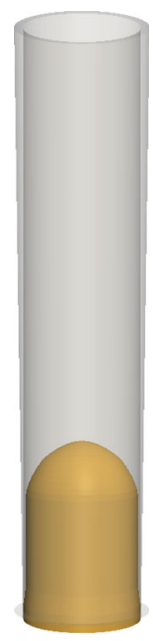

(a)

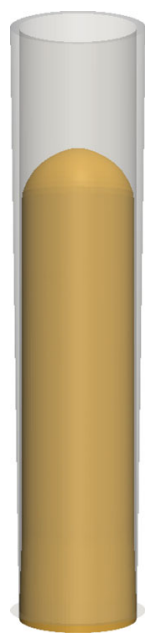

(b) $\delta t=\min \left\{\delta t_{r}, \delta t_{z}, \delta t_{\mathrm{visc}}\right\}$.

Details of the procedure for calculating the time-step size using equations (16a) and (16b) have been given in previous publications by the authors $[27,28]$ and are not presented here.

\section{Tube filling with a polymeric solution}

In order to illustrate the capabilities of the numerical methodology described in this paper, the transient filling of an empty rounded tube was simulated using four meshes: MESH 1: $10 \times 100$ cells $(\mathrm{d} r=\mathrm{d} z=0.1)$, MESH 2: $20 \times$ 200 cells $(\mathrm{d} r=\mathrm{d} z=0.05)$, MESH 3: $40 \times 400$ cells $(\mathrm{d} r=\mathrm{d} z=0.025) \quad$ and $\quad$ MESH $4: \quad 80 \times \quad 800 \quad$ cells $(\mathrm{d} r=\mathrm{d} z=0.0125)$. With the tube empty at time $t=0$,

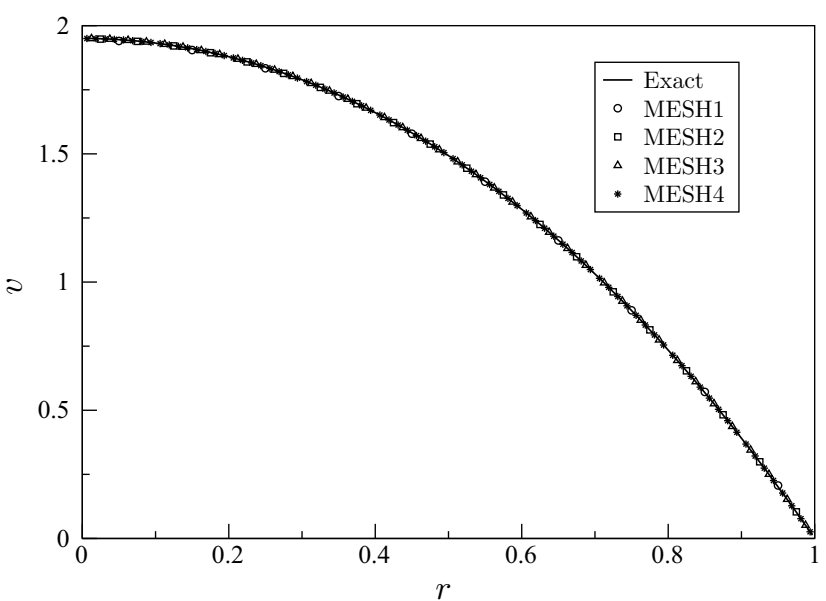

(c)



(d)

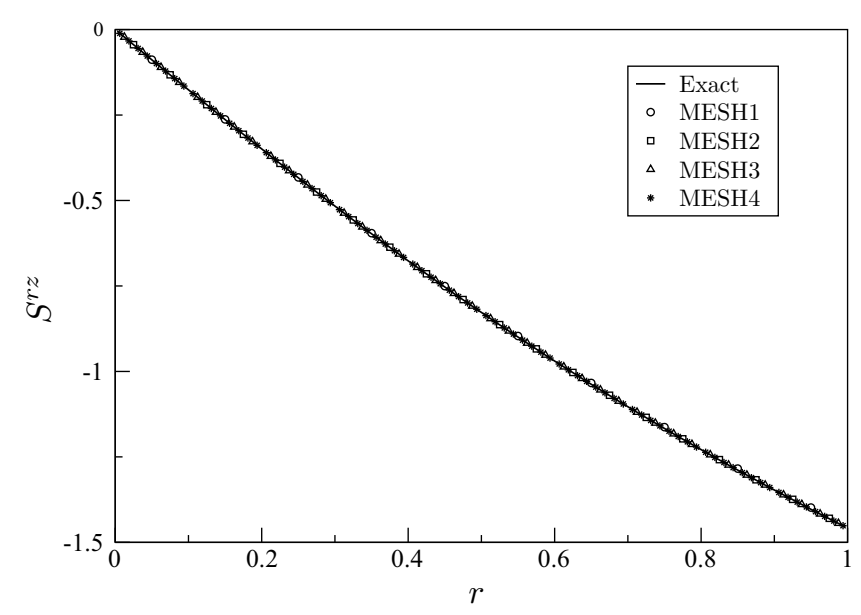

(e)

Fig. 4 Filling of a cylindrical tube with a PTT fluid: $R e=1, W i=0.5, \beta=0.5, \varepsilon=0.1$ and $\xi=0$. a, b $3 \mathrm{D}$ frontal visualizations of the flow at $t=2$ and $t=6$, respectively, on MESH 2. c-e Numerical and exact solutions of $v, S^{z z}, S^{r z}$ at the middle of the tube, $0 \leq r \leq 1, z=5$, at $t=50$ 
fluid was injected in through the tube entrance until it became completely full and steady state was established. The input data employed were: tube radius $L=1 \mathrm{~m}$, tube length $10 L, R e=1, W i=0.5, \beta=0.5, \varepsilon=0.1$ and $\xi=0$. At the tube entrance, the boundary conditions were specified by the PTT-exact solution derived by Cruz et al. in [8]. In dimensionless form, this exact solution can be expressed as:

Table 1 Relative errors between exact and numerical solutions on each mesh

\begin{tabular}{llll}
\hline & $E(v)$ & $E\left(S^{z z}\right)$ & $E\left(S^{r z}\right)$ \\
\hline MESH 1 & $2.71 \mathrm{e}-03$ & $7.15 \mathrm{e}-03$ & $3.54 \mathrm{e}-03$ \\
MESH 2 & $6.90 \mathrm{e}-04$ & $1.80 \mathrm{e}-03$ & $8.96 \mathrm{e}-04$ \\
MESH 3 & $1.73 \mathrm{e}-04$ & $4.52 \mathrm{e}-04$ & $2.24 \mathrm{e}-04$ \\
MESH 4 & $4.34 \mathrm{e}-05$ & $1.13 \mathrm{e}-04$ & $5.62 \mathrm{e}-05$
\end{tabular}



Fig. 5 Decrease of the errors as a function of the mesh spacing



(a)

$$
\begin{aligned}
v(r)= & -\frac{R e}{4 \beta} p_{z}\left(1-r^{2}\right) \\
& +\frac{3 \epsilon R e^{3} W i^{2}}{(1-\beta)^{3} p_{z}}\left[F^{+}(1) G^{-}(1)-F^{+}(r) G^{-}(r)\right. \\
& \left.+F^{-}(1) G^{+}(1)-F^{-}(r) G^{+}(r)\right], \\
S^{r z}= & F^{+}(r)+F^{-}(r), \\
S^{z z}= & \frac{2 W i R e}{1-\beta}\left(S^{r z}\right)^{2}, \\
u=0, & S^{r r}=0 \text { and } S^{\theta \theta}=0
\end{aligned}
$$

where

$$
\begin{aligned}
F^{ \pm}(r) & =\left(C r \pm \sqrt{A^{3}+(C r)^{2}}\right)^{1 / 3}, \\
G^{ \pm}(r) & =3 C r \pm \sqrt{A^{3}+(C r)^{2}}, \\
A & =\frac{(1-\beta)^{2}}{6 \beta \varepsilon(R e W i)^{2}}, \quad C=\frac{(1-\beta)^{3}}{8 \beta \varepsilon(\text { ReWi })^{2}} p_{z} .
\end{aligned}
$$

For this particular simulation, $p_{z}=-7.07212$ was selected.

As the tube is being filled, a "parabolic-like" front will move through the tube; on this free surface, the stress conditions, given by Eqs. (7) and (8), are imposed while the no-slip conditions are applied on the tube wall. Figure $4 a, b$ displays the 3D frontal visualization of the flow at times $t=2$ and $t=6$, respectively.

Some time after the front has reached the exit, steady state will be attained. Figure $4 \mathrm{c}-\mathrm{e}$ displays the exact and numerical profiles obtained for the velocity and the tensor $\mathbf{S}$ on the tube cross section at $z=5$. We can observe in Fig. 4 that the numerical solutions obtained are in good agreement with the exact solutions given by Eqs. (17)(19). To quantitatively demonstrate the convergence of the

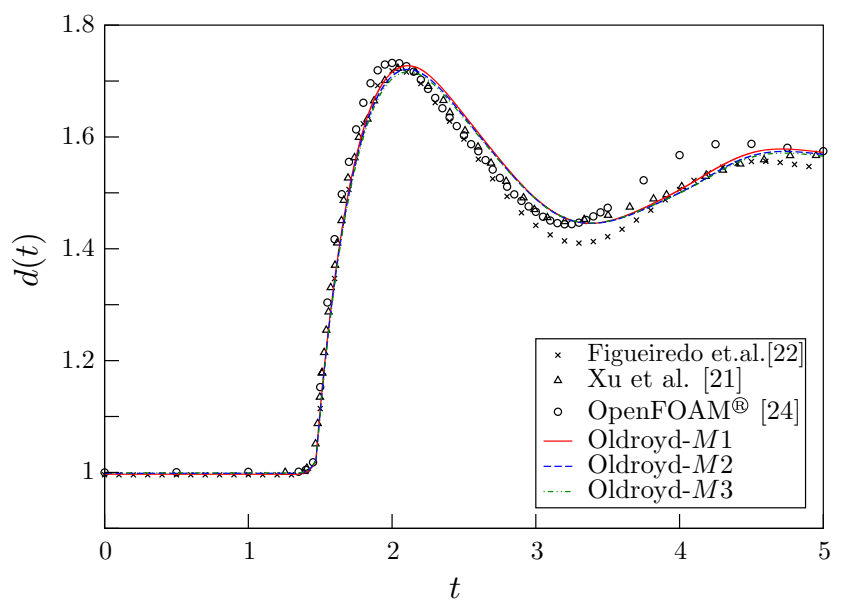

(b)

Fig. 6 a Mesh refinement using the PTT model with $\varepsilon=0.1$ and $\xi=0.2$. Parameters used: $F r=2.26, R e=3, W i=1$ and $\beta=0.1$; b Time evolution of the drop width using an Oldroyd-B fluid and comparisons with results from literature 
Table 2 Input data used in the simulation of drop impacting

\begin{tabular}{lllllll}
\hline$H$ & $R$ (radius) & $U$ & $\rho$ & $\eta_{0}$ & $\lambda$ & $R e$ \\
\hline $0.04 \mathrm{~m}$ & $0.01 \mathrm{~m}$ & $1 \mathrm{~ms}^{-1}$ & $1000 \mathrm{~kg} / \mathrm{s}^{3}$ & $4 \mathrm{~Pa} \mathrm{~s}$ & $0.02 \mathrm{~s}$ & 5 \\
$W i$ & $F r$ & $\varepsilon$ & $\xi$ & $\beta$ & Domain size & Mesh employed \\
1 & 2.26 & 0.1 & 0.2 & 0.1 & $1.25 \times 2.5$ & $100 \times 200$ \\
\hline
\end{tabular}

Fig. 7 3D fluid flow visualization at selected times. Numerical results at $F r=2.26$, $R e=5.0, W i=1, \beta=0.1, \varepsilon=$ 0.1 and $\xi=0.2$

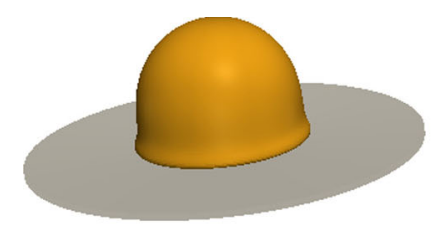

(a) $t=1.5$

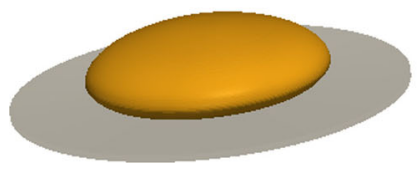

(d) $t=3$

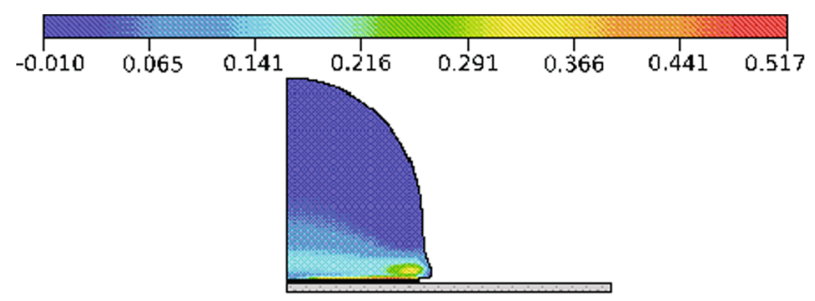

(a) $S^{r r}$

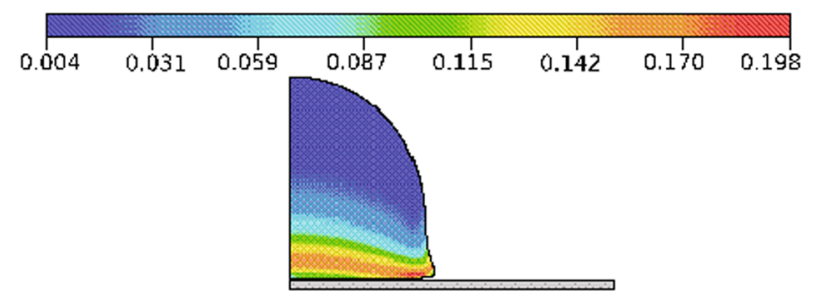

(c) $S^{\theta \theta}$

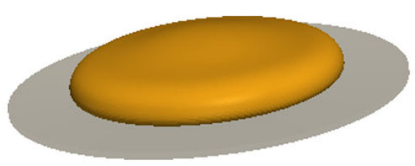

(b) $t=2$



(e) $t=4$

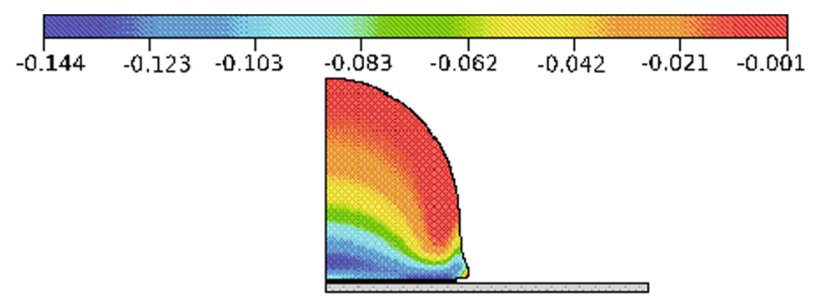

(b) $S^{z z}$

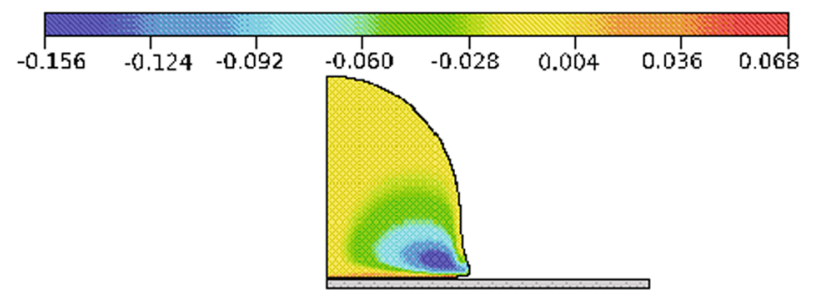

(d) $S^{r z}$

Fig. 8 Profile of the components of the non-Newtonian tensor $\mathbf{S}$ at time $t=1.5$. Numerical results at $F r=2.26, R e=5.0, W i=1, \beta=0.1$, $\varepsilon=0.1$ and $\xi=0.2$

numerical method for this problem, the relative errors $(E(\cdot))$ were calculated on each mesh and are displayed in Table 1 (see also Fig. 5) where we can see that the errors diminish with the mesh spacing. These results indicate convergence of the numerical method.

\section{Simulation of a drop impacting an impermeable surface}

The methodology, described in Section 3, has been implemented in a computer code for viscoelastic free-surface flows. In particular, in this section we shall consider the axisymmetric impacting drop problem described in Section 2.

A drop of radius $R$, falling onto a rigid plate, was simulated. The drop was positioned at a height $H=0.04 \mathrm{~m}$ above the plate with an initial velocity $-U=1.0 \mathrm{~ms}^{-1}$ with gravity acting downwards, i.e., $g=-9.81 \mathrm{~ms}^{-2}$. A $3 \mathrm{D}$ view of the drop and the computational domain are sketched in Fig. 1. 



(b)

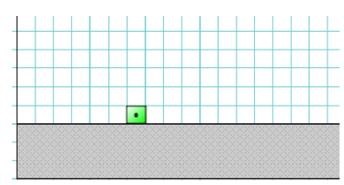

(a)

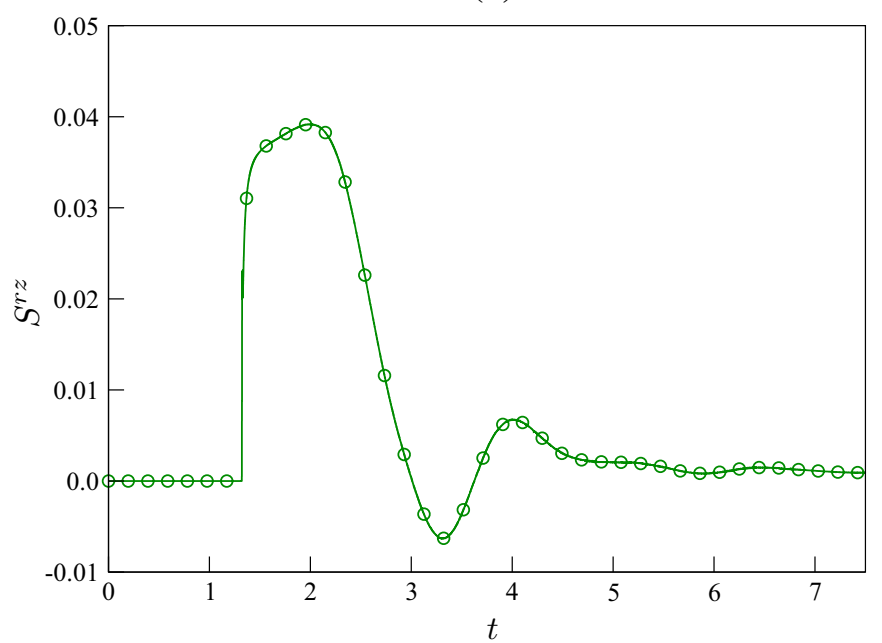

(c)

Fig. 9 b Time evolution of the components of the non-Newtonian tensor $\mathbf{S}$ at the cell adjacent to the rigid boundary, at cell $(i, j)$ highlighted in $(\mathbf{a}$, c) zoom-in on the component $S^{r z}$

\subsection{Mesh refinement and comparison with existing data}

In order to partially verify the correctness of the code, a comparison was made with data from the literature [21, 22] and mesh refinement using the "complete" PTT model was carried out. The impacting drop flow was simulated on three meshes: $M 1(\mathrm{~d} r=\mathrm{d} z=0.0125,100 \times 200$ cells $), M 2$ $(\mathrm{d} r=\mathrm{d} z=0.00625,200 \times 400 \quad$ cells $) \quad$ and $\quad M 3$ $(\mathrm{d} r=\mathrm{d} z=0.003125,400 \times 800$ cells $)$. The nondimensional input parameters were chosen to be $R e=3, W i=1$, $\beta=0.1$ and $F r=2.26$ together with the PTT parameters $\varepsilon=0.1$ and $\xi=0.2$. The results on the three meshes are displayed in Fig. 6a where it is seen that the agreement between the solutions is good. Moreover, in Fig. 6a, it can be seen that the results on mesh $M 1$ are accurate and therefore all the remaining calculations will be performed using this mesh.
With the purpose of comparing the width of the drop, $\mathrm{d}(t)$, with results obtained by other authors using the Oldroyd-B model, one simulation using $R e=5, W i=1$, $\beta=0.1, F r=2.26$ and $\varepsilon=\xi=0.0$ was effected. Figure $6 \mathrm{~b}$ displays $\mathrm{d}(t)$ calculated on mesh $M 1$ together with the solutions of Xu et al. [21], Figueiredo et al. [22] and the OpenFOAM ${ }^{\circledR}$ code [23] for the Oldroyd-B model (see 6b). It can be observed that the results obtained with the code developed in this work are in good agreement with those presented by the above-mentioned authors.

\subsection{Behavior of the non-Newtonian tensor S near the rigid plate}

We are interested in determining the behavior of the nonNewtonian PTT tensor nearby the rigid plate after the drop has impacted it. For this reason, one simulation using the dimensional and nondimensional data summarized in 


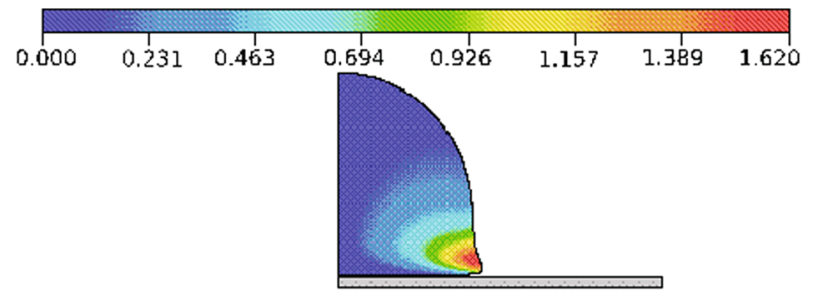

(a) $t=1.5$

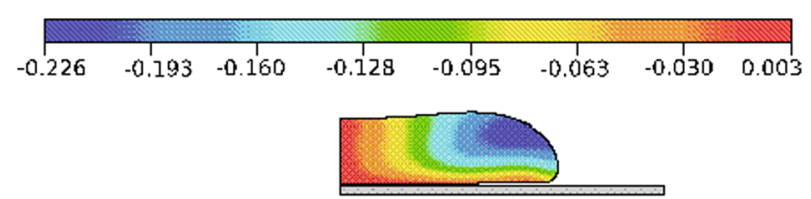

(c) $t=2.5$

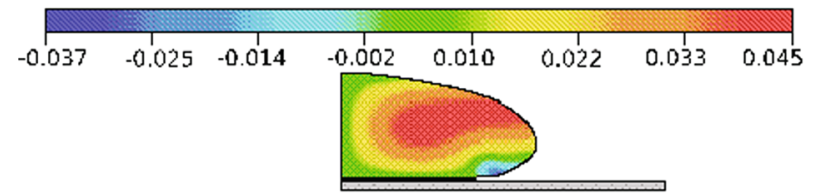

(e) $t=3.5$

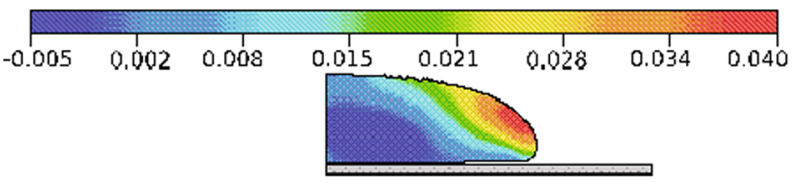

(g) $t=4.5$

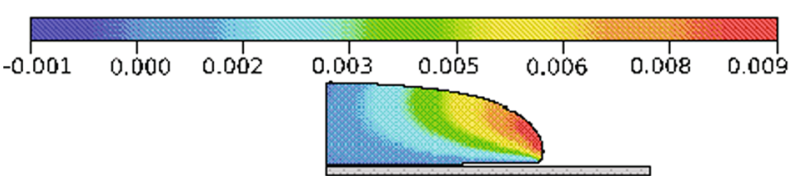

(i) $t=10$


(b) $t=2$

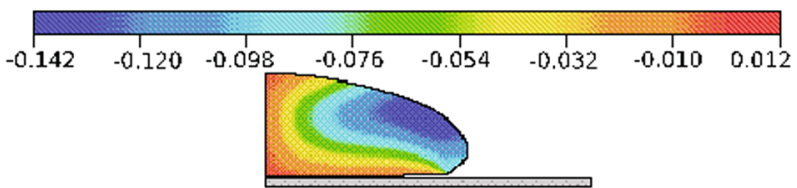

(d) $t=3$

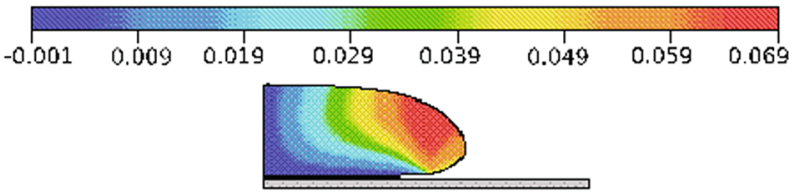

(f) $t=4$

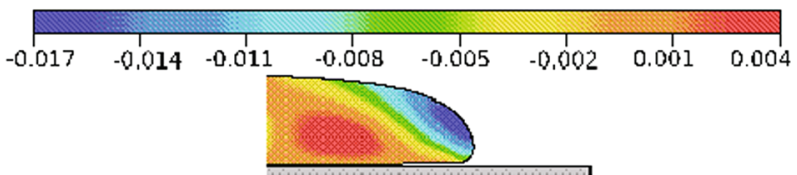

(h) $t=5$

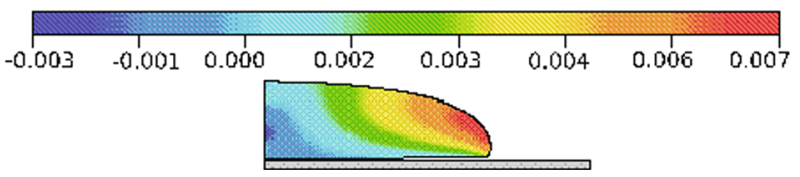

(j) $t=15$

Fig. 10 Profile of the radial $u$-velocity at different times. Numerical results at $F r=2.26, \operatorname{Re}=5.0, W i=1, \beta=0.1, \varepsilon=0.1$ and $\xi=0.2$

Table 3 Parameters used in the simulations of drop spreading flow

\begin{tabular}{llllll}
\hline & $\xi$ & $\varepsilon$ & $\beta$ & $W i$ & $R e$ \\
\hline Reference values & $\mathbf{0 . 1}$ & $\mathbf{0 . 1}$ & $\mathbf{0 . 1}$ & $\mathbf{1}$ & $\mathbf{5 . 0}$ \\
& 0.0 & 0.0 & 0.1 & 0.5 & 3.0 \\
& 0.1 & 0.1 & 0.3 & 1.0 & 5.0 \\
& 0.3 & 0.2 & 0.5 & 1.5 & 7.0 \\
& 0.5 & 0.5 & 0.7 & 2.0 & 10.0 \\
& & & 0.9 & & \\
\hline
\end{tabular}

Table 2 was performed until nondimensional $t=15$. For this particular simulation, the values of the components $S^{r r}, S^{\theta \theta}, S^{z z}$ and $S^{r z}$, at the cell highlighted in Fig. 9a, were monitored in time. Figure 7 displays the 3D fluid flow visualization of the time evolution of the drop deformation along the plate at selected times while Fig. 8 presents the profiles of the components of the non-Newtonian tensor $\mathbf{S}$ at the time $t=1.5$. We can see that soon after the drop collided with the rigid plate, due to the severe deformation caused by the impact, the absolute values of the components of the non-Newtonian tensor $\mathbf{S}$, in regions near to the plate, increased suddenly. To confirm these observations, the time history of the components $S^{r r}, S^{r z}, S^{\theta \theta}$ and $S^{z z}$ at the cell shown in Fig. 9a are plotted in Fig. 9b. We can see that all the components of the tensor $\mathbf{S}$ displayed an overshoot around time $t=1.5$ and then relaxed to zero over time. The shear component (see Fig. 9c) presents several overshoots and oscillates in time during the transient flow. It is also seen that the first normal stress difference $N_{1}=\tau_{z z}-\tau_{r r}$ is always negative. Moreover, Fig. 10 displays several plots showing the time evolution of the velocity component $u$. It is observed that at time $t=1.5$ the velocity on the free surface near the rigid plate becomes very large and positive causing the front surface 


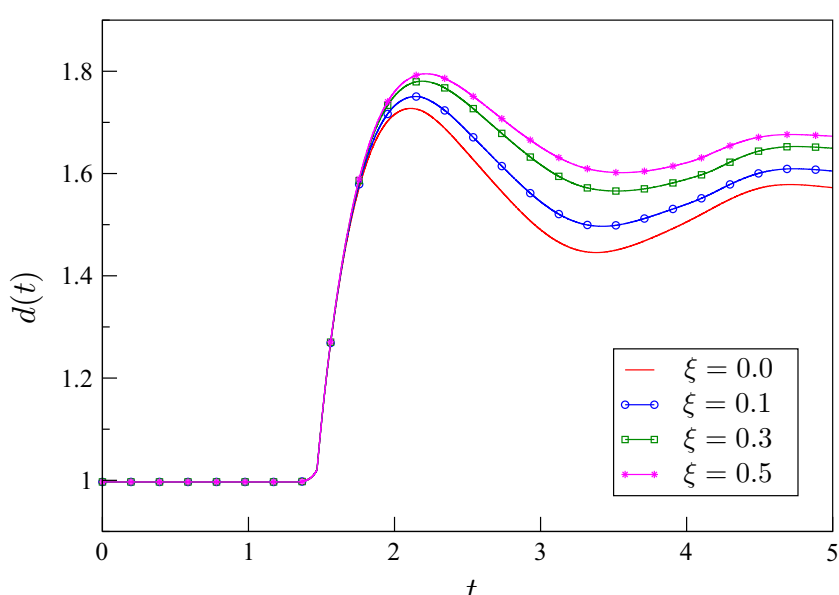

(a)

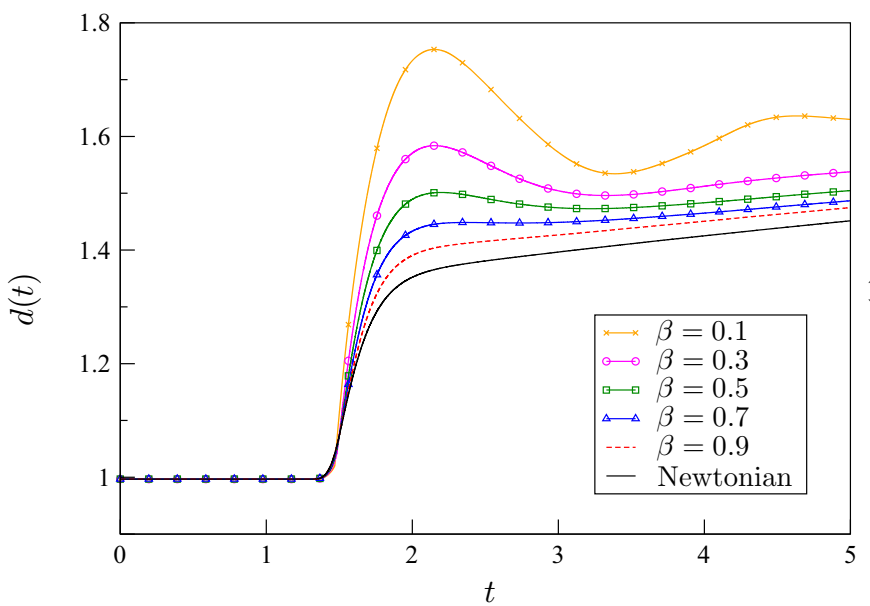

(c)



(b)

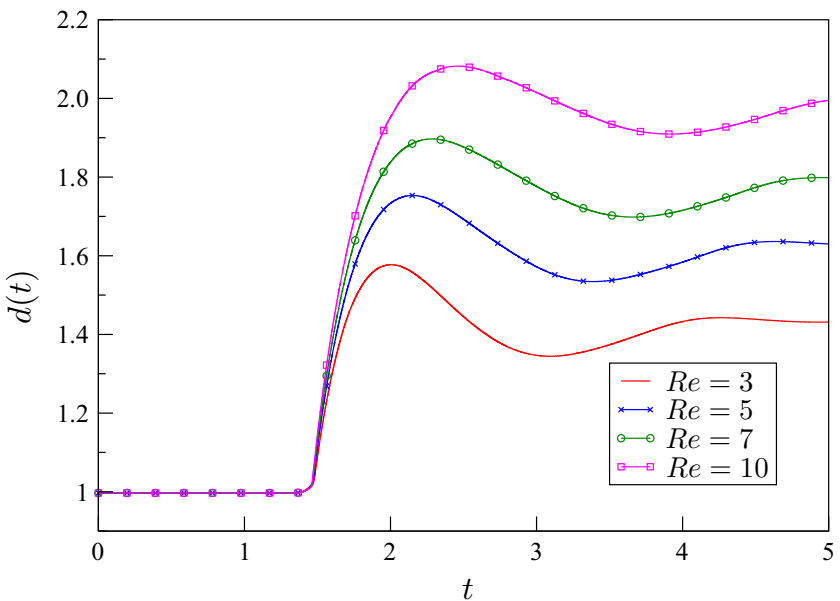

(d)

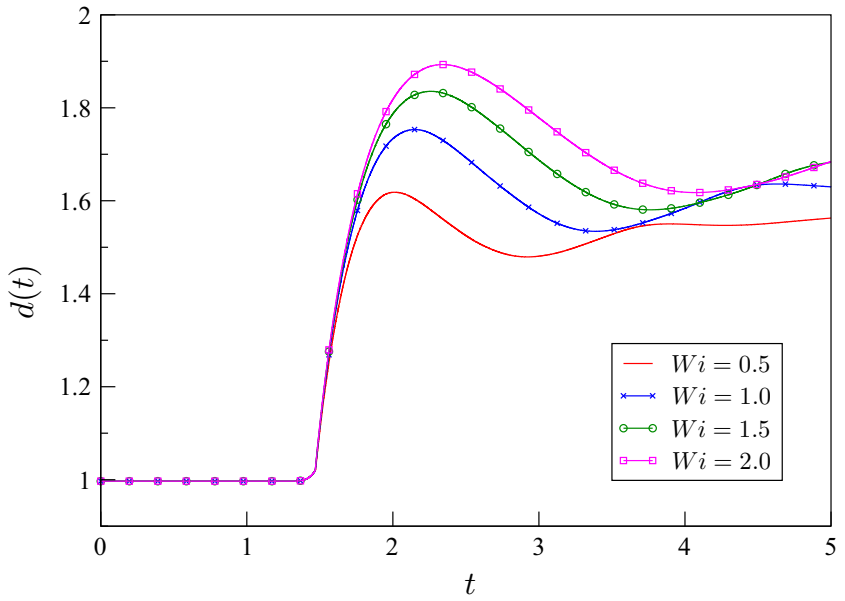

(e)

Fig. 11 Time variation of the width $\mathrm{d}(t)$ of a PTT impacting drop as a function of the nondimensional parameters: $\xi, \varepsilon, \beta, R e$ and $W i$. a Fixed parameters: $R e=5.0, W i=1.0, \beta=0.1, \varepsilon=0.1$. b Fixed parameters: $R e=5.0, W i=1.0, \beta=0.1, \xi=0.1$. c Fixed parameters:
$R e=5.0, W i=1.0, \xi=0.1, \varepsilon=0.1$. d Fixed parameters: $W i=1.0$, $\beta=0.1, \xi=0.1, \varepsilon=0.1$. e Fixed parameters: $R e=5.0, \beta=0.1$, $\xi=0.1, \varepsilon=0.1$ 
to move radially. Later, at times $t=2.5,3$, viscoelasticity within the drop has the effect of making the radial velocity change sign which in turn causes the front surface to retract. At times 3.5, 4, gravitational and inertial forces then make the velocity at the top surface become positive forcing the fluid to move radially. At later times, this cycle is repeated and at time $t=15$, due to viscous and gravitational forces, the velocity is small and the drop is nearly stationary.

\subsection{Drop spreading: an analysis of parameter variation}

To study the viscoelastic effects on the spreading of an axisymmetric drop during the time evolution of the flow, several simulations were performed, varying the parameters that characterize the PTT model, namely, $\xi, \varepsilon, \beta, W i$ and $R e$. In these simulations, the transient width of the drop, $\mathrm{d}(t)$, was plotted as a function of a given parameter of the model. The data employed in this study are displayed in Table 3 where the values highlighted in bold are fixed when a specified parameter is varied, for instance, when the values of the parameter $\xi$ in the first column of Table 3 are used, the values of the parameters $\varepsilon=0.0, \beta=0.1, W i=$ $1, R e=5$ are kept fixed.

Figure 11a displays the results obtained for $\mathrm{d}(t)$ using various values of $\xi$. It can be seen that increasing $\xi$ causes $\mathrm{d}(t)$ to increase. This is in accordance with the PTT model that predicts that the shear viscosity diminishes if $\xi$ increases, providing greater mobility to the free surface. The results obtained with the values of $\varepsilon$ shown in Table 3 are presented in Fig. 11b. It can be observed that the behavior of $\mathrm{d}(t)$ has some similarity with that shown in Fig. 11a. However, the values of $\mathrm{d}(t)$ are larger for larger values of $\varepsilon$. This behavior could possibly have been anticipated since we would expect that elongational viscosity would decrease as $\varepsilon$ increases. The parameter $0<\beta<1$ measures the amount of Newtonian solvent that is added to the polymer. The range varies from pure polymer $(\beta=0)$ to pure Newtonian solvent $(\beta=1)$. Figure $11 \mathrm{c}$ shows the results of $\mathrm{d}(t)$ obtained with the $\beta$ values given in Table 3. We can see that with $\beta=0.1$, the viscoelastic effects are strong and then diminish gradually as $\beta$ increases to $\beta=0.9$, approximating the Newtonian behavior (lowest curve shown with no observed contractions). The influence of the Reynolds number on the diameter of the drop $\mathrm{d}(t)$ is displayed in Fig. 11d where we can observe that, by increasing the Reynolds number, the width of the drop increases. The occurrence of this effect was reported by [14] who used the XPP model and showed that increasing the inertia $(R e)$ led to more mobility for the fluid and consequently increased the spread of the drop. The effect of the variation of Weissenberg number on the drop width is shown in Fig. 11e where it can be seen that increasing the Weissenberg number also causes the drop width to increase. Furthermore, for $W i=1.5,2$, it is noted that steady state has not yet been achieved.

\section{Conclusions}

This work presented a numerical methodology for solving the PTT constitutive equation for time-dependent axisymmetric free-surface flows. The methodology was verified by simulating the flow produced during the filling of a cylindrical tube, and the results were compared with a steady-state analytical solution, showing good agreement. The convergence of the numerical technique was demonstrated by using mesh refinement. The spread of a drop impacting on a rigid surface was simulated. This was compared with different models (using different numerical techniques) employed by a number of investigators; good agreement was displayed. An extensive sensitivity analysis was performed by varying the parameters, $R e, W i, \beta, \epsilon$ and $\xi$, and the results were interpreted physically.

Acknowledgements The authors would like to acknowledge the financial support given by the funding agencies: $\mathrm{CNPq}-$ Conselho Nacional de Desenvolvimento Científico e Tecnológico (Grant No. 306280/2014-0) — and FAPESP_Fundação de Amparo a Pesquisa do Estado de São Paulo [Grants Nos. 11/05090-2 and 2013/07375-0 (CEPID-CeMEAI Project)].

\section{Appendix}

$$
\begin{aligned}
\frac{1}{r} \frac{\partial(r u)}{\partial r}+\frac{\partial v}{\partial z}=0 & \\
\frac{\partial u}{\partial t}+\frac{1}{r} \frac{\partial\left(r u^{2}\right)}{\partial r}+\frac{\partial(u v)}{\partial z}= & -\frac{\partial p}{\partial r}+\frac{\beta}{R e}\left[\frac{1}{r} \frac{\partial}{\partial r}\left(\frac{r \partial u}{\partial r}\right)+\frac{\partial^{2} v}{\partial z^{2}}-\frac{u}{r^{2}}\right] \\
& +\frac{1}{r} \frac{\partial\left(r S^{r r}\right)}{\partial r}+\frac{\partial\left(u S^{r z}\right)}{\partial z}+\frac{S^{\theta \theta}}{r}
\end{aligned}
$$

$$
\begin{aligned}
\frac{\partial v}{\partial t}+\frac{1}{r} \frac{\partial(r v u)}{\partial r}+\frac{\partial\left(v^{2}\right)}{\partial z}= & -\frac{\partial p}{\partial z}+\frac{\beta}{R e}\left[\frac{1}{r} \frac{\partial}{\partial r}\left(\frac{r \partial v}{\partial r}\right)+\frac{\partial^{2} v}{\partial z^{2}}\right] \\
& +\frac{1}{r} \frac{\partial\left(r S^{r z}\right)}{\partial r}+\frac{\partial\left(u S^{z z}\right)}{\partial z}
\end{aligned}
$$

$$
\begin{aligned}
\frac{\partial S^{r r}}{\partial t}= & 2 \frac{(1-\beta)}{R e W i} \frac{\partial u}{\partial r}-\frac{1}{W i} f(\operatorname{Tr}(\mathbf{S})) S^{r r} \\
& -\frac{1}{r} \frac{\partial\left(r u S^{r r}\right)}{\partial r}-\frac{\partial\left(v S^{r r}\right)}{\partial z}+2\left(\frac{\partial u}{\partial r} S^{r r}+\frac{\partial u}{\partial z} S^{r z}\right) \\
& -\xi\left(2 \frac{\partial u}{\partial r} S^{r r}+\left(\frac{\partial u}{\partial z}+\frac{\partial v}{\partial r}\right) S^{r z}\right)
\end{aligned}
$$




$$
\begin{aligned}
\frac{\partial S^{z z}}{\partial t}= & 2 \frac{(1-\beta)}{R e W i} \frac{\partial v}{\partial z}-\frac{1}{W i} f(\operatorname{Tr}(\mathbf{S})) S^{z z} \\
& -\frac{1}{r} \frac{\partial\left(r u S^{z z}\right)}{\partial r}-\frac{\partial\left(v S^{z z}\right)}{\partial z}+2\left(\frac{\partial v}{\partial r} S^{r z}+\frac{\partial v}{\partial z} S^{z z}\right) \\
& -\xi\left(\left(\frac{\partial v}{\partial r}+\frac{\partial u}{\partial z}\right) S^{r z}+2 \frac{\partial v}{\partial z} S^{z z}\right) \\
\frac{\partial S^{r z}}{\partial t}= & \frac{(1-\beta)}{\operatorname{Re} W i}\left(\frac{\partial u}{\partial z}+\frac{\partial v}{\partial r}\right) \\
& -\frac{1}{W i} f(\operatorname{Tr}(\mathbf{S})) S^{r z}-\frac{1}{r} \frac{\partial\left(r u S^{r z}\right)}{\partial r}-\frac{\partial\left(v S^{r z}\right)}{\partial z} \\
& +\frac{\partial v}{\partial r} S^{r r}+\frac{\partial u}{\partial z} S^{z z}+(1-\xi)\left(\frac{\partial v}{\partial z}+\frac{\partial u}{\partial r}\right) S^{r z} \\
& -\frac{\xi}{2}\left(\frac{\partial u}{\partial z}+\frac{\partial v}{\partial r}\right)\left(S^{r r}+S^{z z}\right) \\
\frac{\partial S^{\theta \theta}}{\partial t}= & 2 \frac{(1-\beta) u}{\operatorname{Re} W i} \frac{1}{r} f(\operatorname{Tr}(\mathbf{S})) S^{\theta \theta} \\
& -\frac{1}{r} \frac{\partial\left(r u S^{\theta \theta}\right)}{\partial r}-\frac{\partial\left(v S^{\theta \theta}\right)}{\partial z}+2 \frac{u}{r}(1-\xi) S^{\theta \theta}
\end{aligned}
$$

\section{References}

1. Bird RB, Wiest JM (1995) Constitutive equations for polymeric liquids. Ann Rev Fluid Mech 27:169-193

2. Owens RG, Phillips TN (2002) Computational rheology. Imperial College Press, London

3. Pauli L, Behr M, Elgeti S (2013) Towards shape optimization of profile extrusion dies with respect to homogeneous die swell. J Non-Newton Fluid Mech 200:79-87. https://doi.org/10.1016/ jnnfm.2012.12.002

4. Ngamaramvaranggul V, Webster WF (2001) Viscoelastic simulations of stick-slip and die-swell flows. Int $\mathbf{J}$ Numer Methods Fluids 36:539-595

5. Alves M, Oliveira P, Pinho F (2003) Benchmark solutions for the flow of Oldroyd-B and PTT fluids in planar contractions. J NonNewton Fluid Mech 110:45-75

6. Tomé MF, Grossi L, Castelo A, Cuminato JA, McKee S, Walters K, Die-swell (2007) Splashing drop and a numerical technique for solving the Oldroyd B model for axisymmetric free surface flows. J Non-Newton Fluid Mech 141:148-166

7. Phan-Thien N, Tanner RI (1977) A new constitutive equation derived from network theory. J Non-Newton Fluid Mech 2:353-365

8. Cruz DOA, Pinho FT, Oliveira PJ (2005) Analytical solutions for fully developed laminar flow of some viscoelastic liquids with a Newtonian solvent contribution. J Non-Newton Fluid Mech $132: 28-35$

9. Norouzi M (2017) Analytical solution for the convection of Phan-Thien-Tanner fluids in isothermal pipes. Int $\mathbf{J}$ Therm Sci 108:165-173

10. Konaganti VK, Ansari M, Mitsoulis E, Hatzikiriakos SG (2015) Extrudate swell of a high-density polyethylene melt: II. Modeling using integral and differential constitutive equations. J NonNewton Fluid Mech 235:94-105

11. Oliveira PJ (2003) Asymmetric flows of viscoelastic fluids in symmetric planar expansions geometries. J Non-Newton Fluid Mech 114:33-63
12. Rocha GN, Poole RJ, Oliveira PJ (2007) Bifurcation phenomena in viscoelastic flows through a symmetric 1:4 expansion. J NonNewton Fluid Mech 141:1-17

13. Chilcott M, Rallison JM (1988) Creeping flow of dilute polymer solutions past cylinders and spheres. J Non-Newton Fluid Mech 29:381-432

14. Oishi CM, Martins FP, Tomé MF, Alves MA (2012) Numerical simulation of drop impact and jet buckling problems using the eXtended Pom-Pom model. J Non-Newton Fluid Mech 169-170:91-103

15. Figueiredo RA, Oishi CM, Cuminato JA, Alves MA (2013) Three-dimensional transient complex free surface flows: numerical simulation of XPP fluid. J Non-Newton Fluid Mech 195:88-98

16. Kynch RM, Phillips TN (2017) A high resolution spectral element approximation of viscoelastic flows in axisymmetric geometries using a DEVSS-G/DG formulation. J Non-Newton Fluid Mech 240:15-33

17. Villone MM, D’Avino G, Di Maio E, Hulsen MA, Maffettone PL (2017) Modeling and simulation of viscoelastic film retraction. J Non-Newton Fluid Mech 249:26-35

18. Garduño IE, Tamaddon-Jahromi HR, Walters K, Webster MF (2016) The interpretation of a long-standing rheological flow problem using computational rheology and a PTT constitutive model. J Non-Newton Fluid Mech 233:27-36

19. Mu Y, Zhao G, Chen A, Wu X (2013) Modeling and simulation of tree-dimensional extrusion swelling of viscoelastic fluids with PTT, Giesekus and FENE-P constitutive models. Int J Numer Methods Fluids 72:846-863

20. Ferrás LL, Nóbrega JM, Pinho FT (2012) Analytical solutions for channel flows of Phan-Thien-Tanner and Giesekus fluids under slip. J Non-Newton Fluid Mech 171-172:97-105

21. Xu X, Ouyang J, Jiang T, Li Q (2012) Numerical simulation of 3D-unsteady viscoelastic free surface flows by improved smoothed particle hydrodynamics method. J Non-Newton Fluid Mech 177-178:109-120

22. Figueiredo RA, Oishi CM, Cuminato JA, Azevedo JC, Afonso AM, Alves MA (2014) Numerical Investigation of three dimensional viscoelastic free surface flows: impacting drop problem, Proceedings of 6th European conference on computational fluid dynamics (ECFD VI), TOMO V 5368-5380

23. Jasak H, Jemcov A, Tukovic Z (2007) OpenFOAM: A C++ library for complex physics simulations. In: International workshop on coupled methods in numerical dynamics, IUC Dubrovnik, Croatia, pp 1-20

24. Tomé M, Paulo G, Pinho F, Alves M (2010) Numerical solution of the PTT constitutive equation for unsteady three-dimensional free surface flows. J Non-Newton Fluid Mech 165:247-262

25. Paulo GS, Tomé MF, McKee S (2007) A marker-and-cell approach to viscoelastic free surface flows using the PTT model. J Non-Newton Fluid Mech 147:149-174

26. Batchelor GK (1967) An introduction of fluid dynamics. Cambridge University Press, Cambridge

27. Tomé MF, McKee S (1994) Gensmac: a computational markerand-cell method for free surface flows in general domains. J Comput Phys 110:171-186

28. McKee S, Tomé MF, Ferreira VG, Cuminato JA, Castelo A, Sousa FS, Mangiavacchi N (2008) The MAC method. Comput Fluids 37:907-930

29. Chorin AJ, Marsden JE (2000) A mathematical introduction to fluid mechanics. Springer, New York

30. Tomé MF, Castelo A, Murakami J, Cuminato JA, Minghim R, Oliveira MCF, Mangiavacchi N, McKee S (2000) Numerical simulation of axisymmetric free surface flows. J Comput Phys $157: 441-472$ 